\title{
Calidad nutricional y degradabilidad de tres especies de árboles forrajeros utilizando vacas fistuladas
}

Determination of the nutritional quality and degradability of fodder trees

\author{
species using fistulated cows
}

\author{
Fino Jaime Alfonso ${ }^{1}$, Muñoz Fredy y ${ }^{1}$ Roa Vega María Ligia ${ }^{2}$ \\ ${ }^{1}$ Médico Veterinario Zootecnista. Universidad de los Llanos \\ ${ }^{2}$ Zootecnista, Esp. MSc. Docente Ciencias Animales. Universidad de los Llanos \\ mroa@unillanos.edu.co
}

Recibido 11 de Febrero 2013, Aceptado 15 de Abril 2013

\section{RESUMEN}

Esta investigación se realizó en la granja de la Universidad de los Llanos, Villavicencio Meta, con el objeto de determinar la calidad nutritiva y el potencial de uso en la alimentación animal del Sauce (Salix humboldtiana willd), el chocho (Erythrina rubrinervia) y el canelo (Maytenus laxiflorus), se utilizaron tres novillas cruzadas con Pardo Suizo ruminofistuladas. Estos animales fueron aleatorizados en un diseño de cuadrado latino de sobrecambio simple $3 \times 3$. Los tratamientos consistían en suplementar diariamente con tres kilogramos de materia seca de hojas de estas tres especies adicionando melaza. La dieta basal consistió en suministrar henolaje de kikuyo (Pennisetum clandestinum) a voluntad. En la prueba de degradación se utilizaron bolsas de nylón de $20 \times 10 \mathrm{~cm}$, con un poro promedio de 40 micras aproximadamente. Se colocaron 5 gramos de hojas secas molidas de cada especie de árbol y del henolaje de $P$. clandestinum. Se valoró la degradabilidad ruminal in situ en rumen: de la materia seca (DMS), de la fibra detergente neutro (DFDN) y del nitrógeno total (DNT) a las 6, 12, 24, 48 y 72 horas. En el líquido ruminal se hicieron mediciones de $\mathrm{pH}$ y concentración de nitrógeno amoniacal $\left(\mathrm{N}-\mathrm{NH}_{3}\right)(\mathrm{mg} / 100 \mathrm{ml})$ a las $0,4,8$ y 12 horas. El follaje $M$. laxiflorus presentó una DMS y DFDN más elevadas $(\mathrm{P}<0.05)$, seguido de $S$. humboldtiana, siendo las más bajas las de E. rubrinervia $(P<0.05)$. Las tres especies arbóreas presentaron DMS, DFDN y DNT superiores a los encontrados 
en el henolaje de $P$. clandestinum., $S$. humboldtiana presentó numéricamente una DNT más alta (84.38\%) a las 72 horas de incubación con relación a los otros dos tratamientos. El pH ruminal a diferentes horas siempre se mostró ligeramente básico siendo a las 0 horas, cuando se acercó más a la neutralidad. La concentración de $\mathrm{N}-\mathrm{NH}_{3}(\mathrm{mg} / 100 \mathrm{ml})$ presentó niveles que garantizan un ambiente favorable para la microflora ruminal, y éstos aumentaron después de la ingestión de alimento a las 4 horas observándose una mayor concentración $(P<0.05)$, en el tratamiento con $E$. rubrinervia ( $18.67 \mathrm{ml} / 100 \mathrm{ml}$ de líquido ruminal), mientras que sus tasas de degradación (\% de material/hora) de la MS y FDN fueron las más bajas $(P<0.05)$. La tasa de NT fue inferior para M. laxiflorus $(P<0.05)$. Se concluye que los tres árboles del estudio presentaron buenas respuestas en la mayoría de las variables estudiadas, siendo mejor el comportamiento del $M$. laxiflorus en lo referente a la DMS y DFDN, por lo tanto, estas tres especies, tienen un gran potencial forrajero, para ser utilizados en la alimentación de rumiantes.

Palabras clave: Nutrición de rumiantes, árboles forrajeros.

\section{ABSTRACT}

This research was conducted at the farm of the Universidad de los Llanos, Villavicencio Meta, in order to determine the nutritional quality and potential use in animal feeding: Salix humboldtiana willd, Erythrina rubrinervia and Maytenus laxiflorus) used three crossed with Brown Swiss cows rumen fistulated. These animals were randomized Latin square design single overshift $3 \times 3$. The treatments consisted of providing daily as food suplemento three kilograms of dry matter of crushed leaves of three species adding molasses. The basal diet consisted of grass Pennisetum clandestinum haylage. In degradation test was used nylon bags of $20 \times 10 \mathrm{~cm}$ with an average pore 40 microns. The bags were filled with 5 grams of ground dried leaves of each tree species and $P$. clandestinum haylage. Ruminal degradability was measured in situ on rumen dry matter (DMD) of neutral detergent fiber (NDFD) and total nitrogen (DNT) at 6, 12, 24, 48 and 72 hours. In rumen fluid $\mathrm{pH}$ measurements were made and the concentration of ammonia nitrogen $\left(\mathrm{N}-\mathrm{NH}_{3}\right)(\mathrm{mg} / 100 \mathrm{ml})$ at $0,4,8$ and 12 hours. The foliage $M$. 
laxiflorus presented a higher DMD and NDFD $(P<0.05)$, followed by $S$. Humboldt, the lowest being those of $E$. rubrinervia $(P<0.05)$. The three tree species had $D M D$, NDFD and DNT higher than those found in $P$. clandestinum haylage. $S$. humboldtiana DNT presented numerically one higher (84.38\%) after 72 hours of incubation in relation to the other two treatments. The ruminal $\mathrm{pH}$ at different times was always slightly basic being at 0 hours, when moved closer to neutrality. The $\mathrm{N}$ $\mathrm{NH}_{3}$ concentration $(\mathrm{mg} / 100 \mathrm{ml}$ ) ensure a favorable environment bacterial flora in rumen, and The $\mathrm{N}-\mathrm{NH}_{3}$ concentration increased after food intake at $4 \mathrm{~h}$ with a greater concentration $(\mathrm{P}<0.05)$, in the treatment E. rubrinervia $(18.67 \mathrm{ml} / 100 \mathrm{ml}$ rumen fluid), while their degradation rates (\% material/hour) of DM and NDF were lower $(P<0.05)$. NT degradation rate was lower for $M$. laxiflorus $(P<0.05)$. That the three trees in the study had good responses in most of the variables studied, being better the behavior of $M$. laxiflorus regarding NDFD the DMS and, therefore, these three species have great potential fodder for use in ruminant feed.

Keywords: Ruminant nutrition, fodder trees.

\section{INTRODUCCIÓN}

Los árboles forrajeros son un recurso natural importante que en varias regiones del mundo se vienen utilizando para la alimentación de los animales en sistemas silvopastoriles, sin embargo no se ha investigado lo suficiente, puesto que todavía no están terminados los inventarios de las especies que existen en los bosques y selvas tropicales, donde se encuentra gran variedad de árboles con buen contenido de proteína y con potencial forrajero para la alimentación bovina en diferentes zonas de Colombia. Esto ha despertado en el ganadero gran expectativa por conocerlos y así aumentar la capacidad de carga (Roa, 2004).

Esta investigación estuvo encaminada a caracterizar nutricionalmente el sauce (Salix humboldtiana willd) (Figura 1), el chocho (Erythrina rubrinervia) (Figura 2) y el canelo (Maytenus laxiflorus), que por observaciones de campo, el ganado lo come con avidez, por lo tanto se pueden utilizar como forraje para la alimentación de bovinos, puesto que su digestión en el rumen se caracteriza por procesos 
físicos, bioquímicos y microbiológicos que tienen la capacidad para degradar nutrientes que se encuentran en estos árboles. También se ha demostrado que el tipo de dieta afecta el ambiente del rumen alterando algunos parámetros como es el $\mathrm{pH}$ el cual influye en la degradabilidad del contenido y de la pared celular de los forrajes, (Rosales, 1999; Cordero y Boshier, 2003).

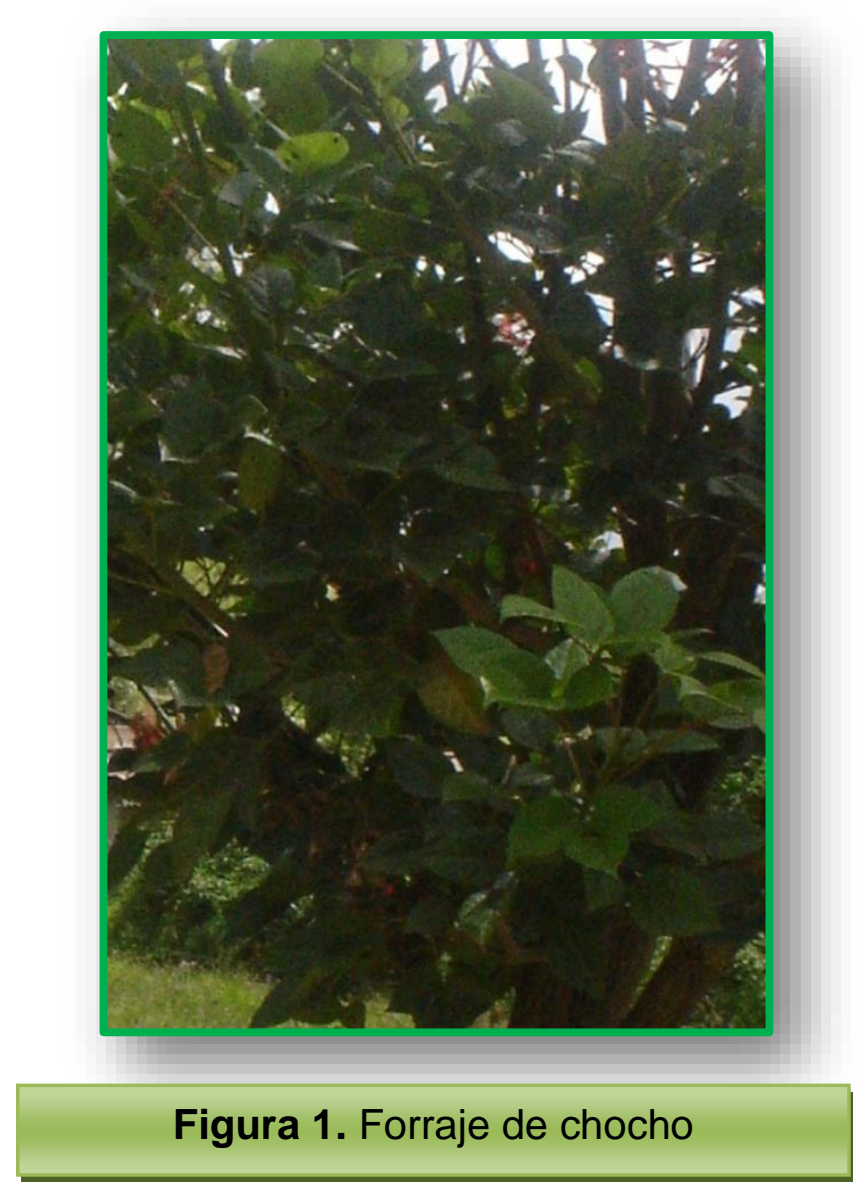

En nueve especies de sauce el contenido de proteína cruda varió apreciablemente desde $15.5 \%$ a $23.7 \%$, los resultados sobre la digestión de proteína cruda in-situ se encuentran enmarcados entre el $57 \%$ y $65.1 \%$. La degradación de la proteína cruda de varias especies de sauce por microorganismos ruminales es más baja que la degradación del heno de gramíneas $(70 \%)$, seguramente varias especies de sauce contienen cantidades considerables de proteína cruda resistentes a la degradación en el rumen, (Ciszuk y Murphy, 1982; Rossi et al., 2005). 


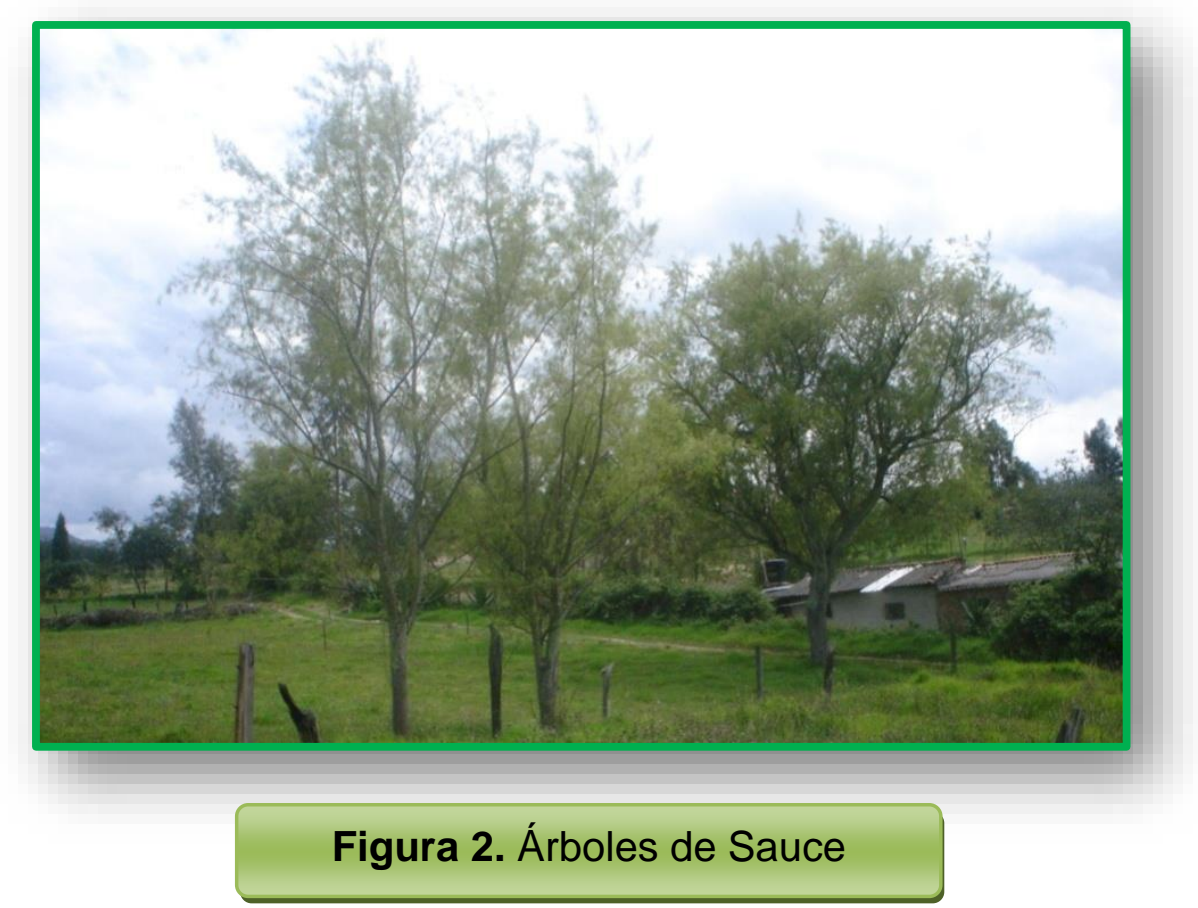

Respecto a los árboles forrajeros que se investigaron en este trabajo se reporta que el sauce (Salix humboldtiana willd) ha sido utilizado como alternativa de alimentación para ovinos y bovinos durante periodos de sequía, a los que se les suministra las ramas grandes de los árboles, y son los mismos animales que la deshojan en pequeñas ramas para facilitar su consumo. Cuando se suministran varias especies de sauce en trozos pequeños de diámetro, se ha observado que se puede mejorar su aprovechamiento, lo cual puede proveer considerablemente los requerimientos de mantenimiento para ovinos y caprinos (Murgueitio e Ibrahim, 2008).

En Nueva Zelanda, concluyeron que algunas especies de Sauces crecidos durante la primavera y el verano podrían ser usados en la alimentación suplementaria durante sequías estivales en alimentación de cabras, seguido por ciervos y por último ovejas que son las menos eficientes. También concluyeron que las concentraciones altas de lignina y taninos condensados limitan el valor nutritivo del sauce (Mc Cabe et al., 1988).

En otro estudio Negi et al., (1990) con terneros ruminofistulados, alimentados con una mezcla de concentrado, más salvado de cereal y sauce a voluntad, 
determinaron que la degradabilidad ruminal de la materia seca y nitrógeno del sauce realizada por la técnica in situ a las 72 horas de degradación fue de $63.2 \%$ y $40.8 \%$, respectivamente. También se analizaron algunos componentes del sauce proteína $12.3 \%$, FDA $35.6 \%$, celulosa $13.3 \%$, lignina $19.1 \%$ y taninos totales 64.4 $\mathrm{g} / \mathrm{kg}$ de materia seca.

El chocho (Erythrina rubrinervia) de la familia de las leguminosas tiene más de 100 especies distribuidas en todo el mundo tropical y subtropical, desde hace muchos años, especies de éste género han participado en subsistemas agrícolas tradicionales como sombrío de café y cacao, cercas vivas, áreas de rotación de cultivos en las selvas húmedas, también se han utilizado sus frutos en la alimentación humana. Flores et al., (1998) y Rossi et al., (2005); reportaron que el género Erythrina presenta hasta un $29.2 \%$ de proteína cruda, $58.5 \%$ de FDN, $38.8 \%$ de FDA y un $54.3 \%$ de digestibilidad in vitro de la materia seca. Benavides, (1986) encontró que a nivel de laboratorio el follaje de especies del género Erythrina se caracterizan por su elevado contenido de proteína cruda (22.7 a $25.4 \%$ ) y mediano de energía digestible (2.16 a $2.42 \mathrm{Mcal} / \mathrm{kg}$. MS) duplicando y triplicando los valores comúnmente reportados para los pastos; sin embargo su nivel de digestibilidad in vitro de la materia seca es medio y similar al de los pastos tropicales (49.0 a $55.4 \%)$.

Roa et al., (1999) evaluaron el potencial nutricional de algunas especies de Erythrina, utilizando tres vacas cebú por criollo rumino-fistuladas, suplementadas diariamente con un $\mathrm{kg}$ de materia seca de estas especies y encontraron que la degradabilidad ruminal de la materia seca fue de $30.9 \%$ a las 6 horas, $39.4 \%$ a las 24 horas y $44.2 \%$ a las 72 horas, la degradabilidad ruminal de la fibra detergente neutro fue de $4.6 \%, 19.1 \%$ y $26.6 \%$ y de la degradabilidad ruminal del nitrógeno total fue de $33.5 \%, 49.6 \%$ y $58.9 \%$, respectivamente a las mismas horas de incubación. En el canelo (Maytenus laxiflorus) no se encuentran publicaciones de experimentos hechos con este género para la alimentación de rumiantes. 


\section{METODOLOGÍA}

La investigación se realizó en la granja y en el Laboratorio de Nutrición y Alimentación animal de la Universidad de los Llanos, localizada en la ciudad de Villavicencio (Meta), altitud de 465 m.s.n.m, temperatura promedio de $27^{\circ} \mathrm{C}$, rango promedio de precipitación anual entre 1.900 y $2.300 \mathrm{~mm}$ y humedad relativa del $80 \%$. Se utilizaron tres (3) vacas cruzadas con Pardo Suizo, fistuladas a nivel del rumen con peso promedio de $350 \mathrm{~kg}$, manejadas en establo, que fueron alimentadas con henolaje de kikuyo (Penisetum clandestinum), sal y agua a voluntad y forraje de árbol. Se hizo un periodo pre-experimental de 10 días, para adaptar a los animales del experimento al manejo rutinario del establo, al consumo del henolaje de kikuyo y a las tres especies de árboles a estudiar.

Previamente al experimento se cortaron ramas con hojas, peciolos y tallos tiernos de árboles adultos de sauce (Salix humboldtiana willd), chocho (Erythrina rubrinervia) y canelo (Maytenus laxiflorus), que se pusieron a secar en latas de zinc al aire libre. Después de seco el forraje, se mezclaron las ramas de cada árbol y fueron molidas homogenizadas para luego ser suministradas a los animales. El diseño del experimento se realizó mediante un cuadrado latino $3 \times 3$ con tres tratamientos, tres periodos y tres animales fistulados en el rumen. Las vacas se rotaron para cada periodo pasando por los tres tratamientos. La duración de cada periodo experimental fue de 14 días, en el cual los primeros 10 días fueron de adaptación a la dieta y los otros 4 días fueron de toma de muestras, los tratamientos fueron: Tratamiento 1: henolaje de kikuyo a voluntad y tres kilogramos de forraje seco de sauce, Tratamiento 2: henolaje de kikuyo a voluntad y tres kilogramos de forraje seco de chocho y Tratamiento 3: henolaje de kikuyo a voluntad y tres kilogramos de forraje seco de canelo. En cada tratamiento a los animales se les ofreció agua y sal mineralizada a voluntad, además a todos los tratamientos se les agregó 300 gramos de melaza para garantizar el consumo.

Las pruebas de degradación in-situ se llevaron a cabo siguiendo la metodología propuesta por Mehrez y Orskov, (1977), se utilizaron bolsas de dacrón de 20 x 10 $\mathrm{cm}$ con un poro promedio de 40 micras aproximadamente. Las bolsas se secaron 
previamente a $60^{\circ} \mathrm{C}$ por 24 horas con el fin de colocarlas a peso constante. En cada bolsa se colocaron 5 gramos de muestra seca de tratamiento por duplicado para cada tiempo. Las incubaciones en el rumen se realizaron a las $6,12,24,48$ y 72 horas, se midió degradabilidad ruminal de la materia seca (DMS), degradabilidad ruminal de fibra detergente neutro (DFDN) y degradabilidad ruminal de nitrógeno total (DNT). En el líquido ruminal se realizaron mediciones de $\mathrm{pH}$ y nitrógeno amoniacal a las $0,4,8$ y 12 horas. El henolaje de kikuyo y los forrajes secos de sauce, chocho y canelo, fueron sometidos a un análisis preliminar en el cual se determinó a cada uno, materia seca (MS), extracto etéreo (EE), nitrógeno total (NT), cenizas, fibra detergente neutro (FDN), con cuatro repeticiones por muestra con el fin de conocer parcialmente la caracterización bromatológica que posee cada tratamiento antes de medir su degradabilidad en el rumen, siguiendo la metodología de la organización americana de química analítica (AOAC, 2005) y Van Soest et al., 1991. El análisis estadístico fue un cuadrado latino $3 \times 3$. Las variables a analizar fueron las tasas de degradación a diferentes horas de MS, FDN, NT de los tratamientos, y en el líquido ruminal el $\mathrm{pH}$ y $\mathrm{N}-\mathrm{NH}_{3}$. Se utilizó la prueba de Duncan para la comparación de medias. Los valores de degradabilidad fueron ajustados por el modelo de Mehrez y Orskov, (1977) según ecuación:

$\mathrm{D}=\mathrm{a}+\mathrm{b}\left(1-\mathrm{e}^{-\mathrm{ct}}\right)$

"D"= Degradación de la fracción soluble en un determinado tiempo (t).

"a"= Intercepto de la curva en el tiempo cero.

"b"= Potencia de degradabilidad cuando el tiempo no es limitado.

"c"= Tasa de degradación constante de la fracción "b" por hora.

"e"= Logaritmo natural.

\section{RESULTADOS Y DISCUSIÓN}

Los análisis proximales (Tabla 1) permitieron observar que el forraje con mayor valor de proteína cruda (PC) es el chocho (Erythrina rubrinervia) con un $23.13 \%$, seguido del sauce (Salix humboldtiana) con un $19.35 \%$ y por último el canelo (Maytenus laxiflorus) con un $8.4 \%$; al compararlos con el porcentaje de proteína cruda del henolaje de kikuyo que es $9.37 \%$, se observa que el chocho y el sauce lo superan en más del doble de su valor, y el canelo tiene un porcentaje menor. 
Tabla 1. Composición (\%) nutricional de follajes de sauce, chocho, canelo y henolaje de kikuyo

\begin{tabular}{lcccc}
\hline \multicolumn{1}{c}{ Nutriente } & Sauce & Chocho & Canelo & Henolaje \\
\hline Materia seca & 23 & 20 & 28 & 52 \\
Humedad & 77 & 80 & 72 & 48 \\
Proteína cruda & 19.35 & 23.13 & 8.4 & 9.37 \\
Fibra cruda & 15.13 & 21.63 & 15.29 & 25.43 \\
Fibra detergente Neutro & 55.47 & 51.16 & 64.87 & 62.45 \\
(FDN) & 1.17 & 2.46 & 3.69 & 1.67 \\
Extracto etéreo & 5.42 & 9.8 & 8.11 & 5.95 \\
Cenizas & 62.24 & 47.11 & 64.85 & 54.58 \\
Extracto no nitrogenado &
\end{tabular}

Muestras analizadas en el Laboratorio de Nutrición Animal de UNILLANOS

Comparando con los resultados de Benavides, (1986) quien reportó un valor promedio de proteína cruda de distintas especies de Eritryna entre 22.7 a $25.4 \%$, la Erythrina rubrinervia presenta valores de PC similares a las otras especies de su género; y su valor supera la PC encontrados por Roa et al., (1999) en Erythrina glauca (18.12\%) y Erythrina poeppigiana (21.25\%). Aunque inferiores al valor de PC encontrado por Flores et al. (1998) en Erythrina berteroana (29.2\%).

Los tres forrajes de árboles presentaron altos valores de FDN siendo el más alto el de canelo con $64.87 \%$ seguido del sauce con $55.47 \%$ y por último el chocho con $51.16 \%$. El valor de FDN del henolaje de kikuyo fue $62.45 \%$. Los altos valores encontrados pueden estar correlacionados con la edad de las plantas muestreadas (madurez avanzada) debido a muy pocas o nada de podas (Tabla 1). La Erythrina rubrinervia presentó una FDN ligeramente superior a los valores encontrados por Roa et al., (1999) en Erythrina glauca (51.1\%) y Erythrina poeppigiana (47.5\%), y muy inferior al encontrado por Flores et al., (1998) en Erythrina berteroana (58.5\%).

En la Gráfica 1 se observa que la concentración de $\mathrm{N}^{-\mathrm{NH}_{3}}$ a las 0,8, y 12 horas fue similar entre tratamientos $(P>0.05)$, Roa et al., (2002) mencionan que la 
cantidad de amoniaco que permite una digestión máxima en el rumen y a su vez una población alta de microorganismos varía según la dieta. El nivel crítico se reporta desde 5 a 25 miligramos de nitrógeno amoniacal por 100 mililitros de líquido ruminal; valores que coinciden con los encontrados en este experimento. Sin embargo, Flores et al., (1998) sugieren que concentraciones mínimas de 10-15 miligramos de amonio por 100 mililitros de fluido ruminal pueden garantizar un ambiente favorable en el rumen. Las concentraciones ruminales de amoniaco aumentaron después de la ingestión de alimento a las 4 horas, siendo más elevada $(\mathrm{P}<0.05)$ la concentración en el tratamiento con chocho (18.67 miligramos) seguido del sauce (16.33) y la más baja fue la del canelo (13.07).

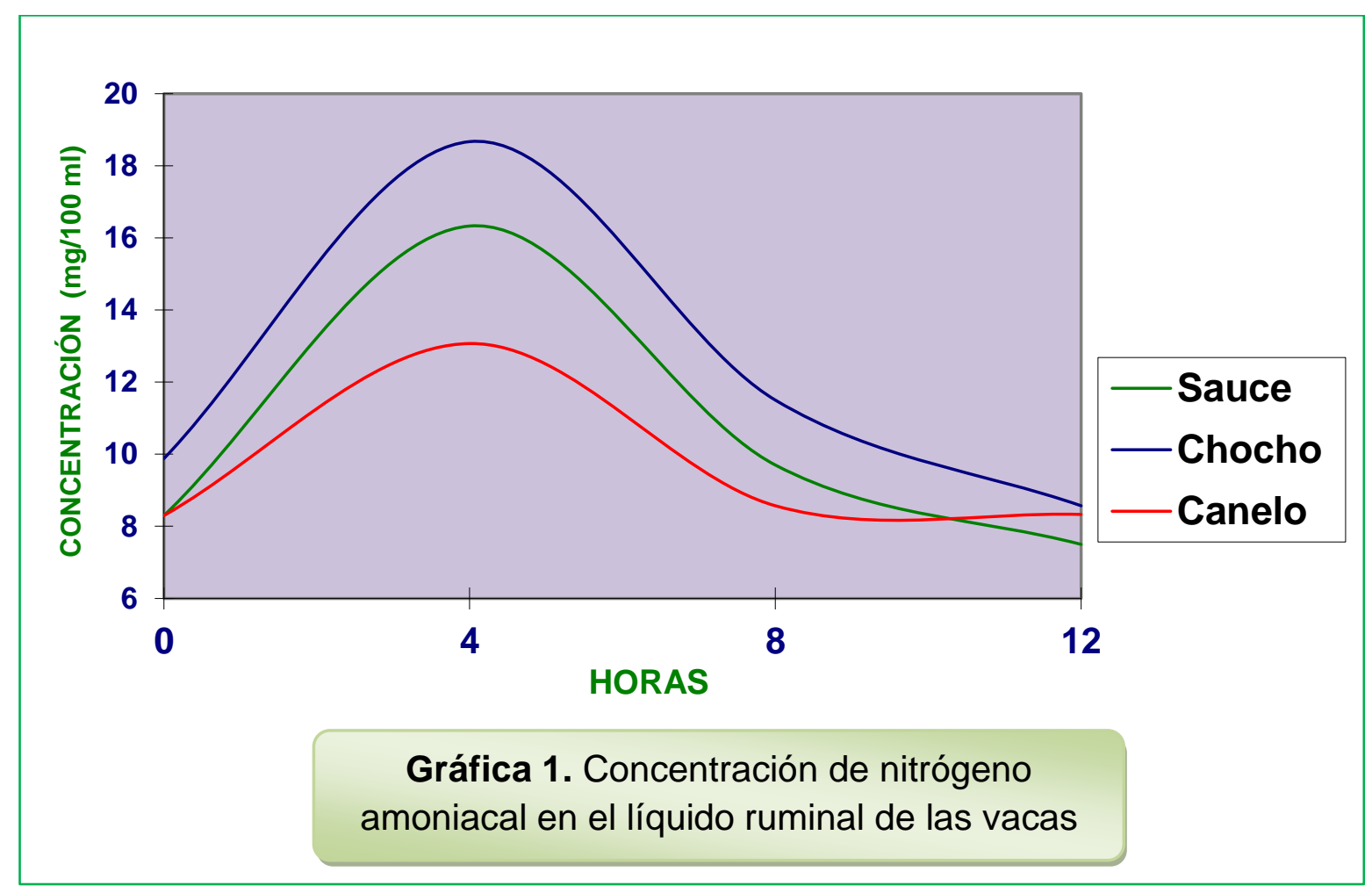

Estos resultados concuerdan con lo mencionado por Owens y Goetsh, (1988) quienes afirman que las concentraciones ruminales de amoniaco aumentan después de la ingestión de alimento y el tamaño del incremento está determinado por la solubilidad de la proteína. A las 8 horas la concentración de $\mathrm{N}-\mathrm{NH}_{3}$ bajó marcadamente en todos los tratamientos (Gráfica 1). 
El pH ruminal a diferentes horas siempre se mostró ligeramente básico, siendo a las 4 horas cuando se acercó más a la neutralidad (Tabla 2). Los datos obtenidos en la medición de $\mathrm{pH}$ a las diferentes horas no presentaron diferencia significativa entre tratamientos, excepto en la hora 0 donde el $\mathrm{pH}$ del tratamiento con sauce fue más alcalino $(\mathrm{P}<0.05)$ en comparación con los demás tratamientos (Tabla 2$)$.

Tabla 2. Valores de $\mathrm{pH}$ del líquido ruminal en los diferentes tratamientos

\begin{tabular}{cccc}
\hline \multirow{2}{*}{ Hora } & \multicolumn{3}{c}{ Tratamientos } \\
\cline { 2 - 4 } & Sauce & Chocho & Canelo \\
\hline 0 & $7.75^{a}$ & $7.51^{b}$ & $7.52^{b}$ \\
4 & $7.11^{a}$ & $7.18^{a}$ & $7.17^{a}$ \\
8 & $7.21^{a}$ & $7.24^{a}$ & $7.24^{a}$ \\
12 & $7.62^{a}$ & $7.45^{a}$ & $7.58^{a}$ \\
\hline
\end{tabular}

Filas con distintas letras son diferentes $(\mathrm{P}<0.05)$

Fuente: el estudio

El canelo presentó la DMS y DFDN más alta $(P<0.05)$ en las diferentes horas en comparación con los otros dos tratamientos: sauce y chocho, excepto la DMS a las 6 y 12 horas que fue similar a la del sauce (Tabla 3). La DMS del chocho fue inferior $(P<0.05)$ en todas las horas a la del sauce, (Tabla 3 ). Igual sucedió con la DFDN del chocho que fue más baja $(\mathrm{P}<0.05)$ que la del sauce en la mayoría de las horas, excepto a las 6 horas que fue similar (Tabla 3). La DNT presentó un comportamiento similar en los tres tratamientos en todas las horas excepto a las 48 horas la DNT de sauce que fue superior $(\mathrm{P}<0.05)$ con relación a los otros dos tratamientos, (Tabla 3). La DMS y DFDN del canelo a las 72 horas es $7.99 \%$ y $15.89 \%$ superior a la DMS y DFDN del sauce, y $17.97 \%$ y $23.54 \%$ a la DMS y DFDN del chocho, respectivamente (Tabla 3 y Grafica 2). La tasa de degradabilidad ruminal de la MS y FDN del chocho fue inferior $(P<0.05)$ en comparación con sauce y canelo, mientras la tasa de degradación del NT fue más baja $(P<0.05)$ en canelo (Tabla 4$)$. 


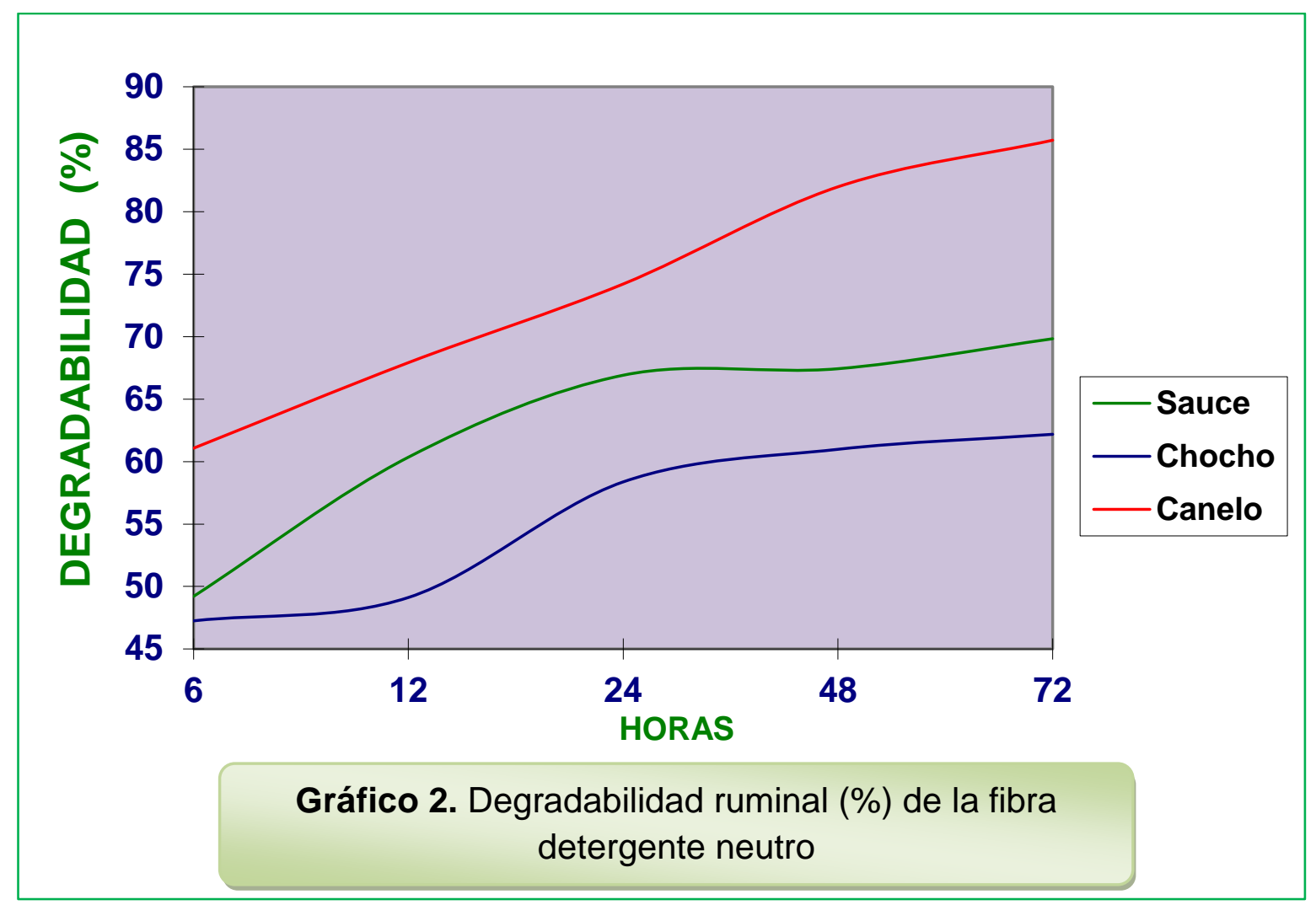

La DMS del sauce (Salix humboldtiana willd) es alta a las 48 horas $(71.05 \%$ ) si se compara con las degradabilidades reportadas por Hathaway (1985), quien encontró degradabilidades de $67.6 \%, 47.6 \%$ y $53.7 \%$ a las 48 horas para el Salix matsudana $\mathrm{X}$ alba, el Salix discolor, y Salix viminalis gigantea respectivamente. Negi et al., (1990) determinó que la DMS del Salix tetrasperma fue $63.2 \%$ a las 72 horas, valor muy bajo comparado con $72.30 \%$ del Salix humboldtiana a la misma hora, que se encontró en este trabajo.

La DMS de canelo (E. rubrinervia) es superior (80.29\%) a las encontradas por Roa et al., (2004) en E. glauca quienes reportaron $52.0 \%$ a las 72 horas. La DMS reportada por Flores et al., (1998) en E. berteroana (54.3\%) y la DMS en distintas especies de Erythrina (49.0 a 55.4\%) también son inferiores a la DMS de $E$. rubrinervia. 
Tabla 3. Degradabilidad ruminal in-situ (\%) de la materia seca, fibra en detergente neutro y nitrógeno total en los diferentes tratamientos

\begin{tabular}{ccccccc}
\hline \multirow{2}{*}{ Forraje } & \multicolumn{5}{c}{ Horas } \\
\cline { 3 - 7 } & & $\mathbf{6}$ & $\mathbf{1 2}$ & $\mathbf{2 4}$ & $\mathbf{4 8}$ & $\mathbf{7 2}$ \\
\hline \multirow{3}{*}{ DMS } & Sauce & $47.94^{a}$ & $58.98^{a}$ & $70.05^{b}$ & $71.05^{b}$ & $72.30^{b}$ \\
& Chocho & $38.69^{b}$ & $46.14^{b}$ & $59.31^{c}$ & $61.45^{c}$ & $62.32^{c}$ \\
& Canelo & $49.81^{a}$ & $58.78^{a}$ & $74.29^{a}$ & $78.98^{a}$ & $80.29^{a}$ \\
& & & & & & \\
\multirow{2}{*}{ DFDN } & & & & & \\
& Sauce & $49.21^{b}$ & $60.35^{b}$ & $66.91^{b}$ & $67.43^{b}$ & $69.83^{b}$ \\
& Chocho & $47.25^{b}$ & $49.12^{c}$ & $58.37^{c}$ & $60.98^{c}$ & $62.18^{c}$ \\
& Canelo & $61.07^{a}$ & $67.92^{a}$ & $74.21^{a}$ & $81.99^{a}$ & $85.72^{a}$ \\
& & & & & & \\
DNT & & & & & \\
& Sauce & $44.10^{a}$ & $58.82^{a}$ & $78.82^{a}$ & $82.42^{a}$ & $84.38^{a}$ \\
& Chocho & $46.59^{a}$ & $56.83^{a}$ & $71.40^{a}$ & $81.42^{a b}$ & $73.72^{a}$ \\
& Canelo & $46.29^{a}$ & $56.97^{a}$ & $74.60^{a}$ & $79.65^{b}$ & $83.79^{a}$ \\
\hline
\end{tabular}

Columnas con distintas letras son diferentes $(P<0.05)$. DMS: Degradabilidad ruminal in-situ de la materia seca. DFDN: Degradabilidad ruminal in-situ de la fibra en detergente neutro. DNT: Degradabilidad ruminal in-situ del nitrógeno total.

Analizando los contenidos de materia seca y sus degradabilidades se puede afirmar que el follaje que más aporta materia seca es el canelo y que su calidad resultó ser la mejor puesto que se degrada casi en su mayoría (80.29\%) a las 72 horas. El sauce es el segundo árbol en contenido de materia seca con un $23 \%$ de la cual se degrada un $72.30 \%$ a las 72 horas. La DFDN del sauce a las 6, 24 y 72 horas de incubación, son inferiores a los $47.25,58.37$ y $62.18 \%$ comparadas con los valores de E. rubrinervia.

El Salix humboldtiana willd muestra valores DNT de $84.38 \%$ a las 72 horas (Tabla $3)$, que son muy superiores con respecto a los valores encontrados por Negi (1990) en Salix tetrasperma quien reportó $40.8 \%$ a las 72 horas. Los valores de DNT del chocho (E. rubrinervia), a las 72 horas $(73.72 \%)$ son superiores a los de E. glauca $56.8 \%$ encontrados por Roa (2004). 
Tabla 4. Tasa de degradación (\% de material/hora) de las tres especies arbóreas estudiadas

\begin{tabular}{cccc}
\hline Parámetro & Sauce & Chocho & Canelo \\
\hline Materia seca & $6.2^{a}$ & $5.5^{b}$ & $6.7^{a}$ \\
FDN & $5.2^{a}$ & $4.3^{b}$ & $5.8^{a}$ \\
NT & $6.3^{a}$ & $5.2^{a}$ & $4.8^{b}$ \\
\hline
\end{tabular}

Filas con letras distintas son diferentes $(P<0.05)$. FDN: Fibra detergente neutro. NT: nitrógeno total. Fuente: El estudio

La DMS y DFDN del henolaje de kikuyo fueron similares, excepto en las 12 horas de incubación de la DFDN (Tabla 5), demostrando que el suministro de follaje de los árboles en estudio no tiene efecto sobre la DMS y la DFDN del henolaje de kikuyo. La DNT del henolaje de kikuyo cuyos resultados oscilan entre $38.25 \%$ hasta $72.85 \%$ desde las 6 hasta las 72 horas, presentó una ligera diferencia significativa entre tratamientos en las primeras 24 horas (Tabla 5). La DMS, DFDN y DNT de los tres árboles en estudio fueron superiores a las del henolaje de kikuyo (Tablas 6 y 8), indicando su potencial como alimento para bovinos (Tablas 3 y 5 ). La DFDN del henolaje se comporta mejor frente al tratamiento con canelo logrando una degradabilidad del $63.62 \%$ a las 72 horas, aunque el porcentaje de degradación de la fibra en sus primeras fases es mucho más veloz en el tratamiento con Sauce $34.23 \%$ y $41.34 \%$ a las 6 y 12 horas respectivamente (Tabla 5).

\section{CONCLUSIONES}

Los forrajes de canelo (Maytenus laxiflorus), sauce (Salix humboldtiana willd) y chocho (Erythrina rubrinervia) presentan altas degradabilidades in situ de MS, FDN y NT, los cuales tienen potencial para ser usados en alimentación de rumiantes. El follaje de canelo presenta una degradabilidad ruminal de materia seca y FDN superior al sauce y este al chocho. La degradabilidad ruminal del nitrógeno total es similar en los tres follajes. 
Tabla 5. Degradabilidad ruminal in situ (\%) de la materia seca, fibra en detergente neutro y nitrógeno total del henolaje de kikuyo en los diferentes tratamientos

\begin{tabular}{|c|c|c|c|c|c|c|}
\hline & \multirow{2}{*}{ orraje } & \multicolumn{5}{|c|}{ Horas de Incubación } \\
\hline & & 6 & 12 & 24 & 48 & 72 \\
\hline \multirow{3}{*}{ DMS } & Hen. Sauce & $30.73^{a}$ & $37.75^{a}$ & $48.31^{a}$ & $57.07^{a}$ & $60.85^{a}$ \\
\hline & Hen. Chocho & $27.19^{a}$ & $35.59^{a}$ & $45.56^{a}$ & $52.32^{a}$ & $54.02^{a}$ \\
\hline & Hen. Canelo & $29.51^{a}$ & $36.32^{a}$ & $47.89^{a}$ & $55.20^{a}$ & $61.07^{a}$ \\
\hline \multirow{3}{*}{ DFDN } & Hen. Sauce & $34.23^{a}$ & $41.34^{a}$ & $50.35^{a}$ & $59.54^{a}$ & $62.20^{a}$ \\
\hline & Hen. Chocho & $27.06^{a}$ & $34.06^{a b}$ & $47.14^{a}$ & $52.19^{a}$ & $56.32^{a}$ \\
\hline & Hen. Canelo & $23.61^{a}$ & $29.66^{b}$ & $46.67^{a}$ & $55.90^{a}$ & $63.67^{a}$ \\
\hline \multirow{3}{*}{ DNT } & Hen. Sauce & $38.25^{b}$ & $43.75^{b}$ & $51.53^{b}$ & $62.61^{a}$ & $64.55^{a}$ \\
\hline & Hen. Chocho & $32.32^{a}$ & $58.14^{a}$ & $66.87^{a}$ & $68.14^{a}$ & $69.76^{a}$ \\
\hline & Hen. Canelo & $46.75^{b}$ & $53.87^{a}$ & $62.03^{a b}$ & $69.32^{a}$ & $7285^{a}$ \\
\hline \multicolumn{7}{|c|}{$\begin{array}{l}\text { Columnas con distintas letras son diferentes }(\mathrm{P}<0.05) \text {. DMS: Degradabilidad ruminal in situ de la } \\
\text { materia seca del henolaje con el respectivo forraje de árbol. } \\
\text { DFDN: Degradabilidad ruminal in situ de la fibra en detergente neutro del henolaje con el } \\
\text { respectivo forraje de árbol. DNT: Degradabilidad ruminal in situ del nitrógeno total del henolaje con } \\
\text { el respectivo forraje de árbol. }\end{array}$} \\
\hline \multicolumn{7}{|c|}{$\begin{array}{l}\text { Los niveles de } \mathrm{N}-\mathrm{NH}_{3} \text { del líquido ruminal fueron similares en los diferentes } \\
\text { tratamientos, y estuvieron dentro de los rangos normales reportados en otras } \\
\text { investigaciones. El pH ruminal se presenta ligeramente básico en los } 3 \\
\text { tratamientos y a todas las horas de medición. Esta investigación básica sobre la } \\
\text { degradabilidad ruminal del follaje de chocho, canelo y sauce, da pautas para } \\
\text { realizar otros trabajos. }\end{array}$} \\
\hline
\end{tabular}

\section{REFERENCIAS BIBLIOGRÁFICAS}

1. A.O.A.C. Official methods of Analytical Association of Official Analytical Chemical. Washington. D.C; 1990. 
2. Benavides, J. E. Utilización del follaje de poró (Erythrina poeppigiana) para alimentar cabras en condiciones de trópico húmedo. En: Congreso de la asociación mexicana de zootecnistas y técnicos de caprinocultura, Mazatlán, México. Memorias: Turrialba, Costa Rica; 23 p. 1986.

3. Ciszuk y Murphy M. Digestion of crude Protein and organic matter of leaves by rumen microbes in vitro. In: swedish J. Agric.12: 35-40. Res 1982.

4. Flores O. I., Bolívar M. A., Botero J. A. Parámetros nutricionales de algunas arbóreas leguminosas y no leguminosas con potencial forrajero para la suplementación de rumiantes en el trópico. Livestock. Research for rural Development. 10: 1: 1-9. 1998.

5. Cordero J., Boshier D. H. (editores). Árboles de Centroamérica. Oxford Forestry Institute Catie. 1080 p. 2003.

6. Hathaway R. L. Short - Rotation coppiced willows for sheep fodder in New Zealand. Agricultural Science. p. 140-142. 1985.

7. Mc Cabe S., Barry T. Nutritive value of willow (Salix $s p$ ) for sheep, goats and deer. Printed in great Britain. J Agric. Sci., 111: 1-9. Camb 1988.

8. Mehrez A. Z., Orskov E. R. A study of artificial bag thecnique for determining the digestibility of feed on the rumen. J. Agr. Sci, Comb 88: 645-650. 1977.

9. Murgueitio E., Ibrahim M. "Ganadería y medio ambiente en América Latina". En: Ganadería del futuro: investigación para el desarrollo. (Murgueitio E., Cuartas C. y Naranjo J. editores) 2008.

10. Negi B., Makkar H. Influence of variable rumen degradation constants determined by different models on effective degradability of dry mattter and nitrogen. Indian J. Anim. Nutr. 7: 4: 235-244. 1990.

11. Nocek J., Grant A L. A characterization of in-situ nitrogen and fiber digestion and baterial nitrogen contamination of hay forages preserved at different dry matter percentages. J. Anim. Sci.; 64. 552-564. 1987

12. Ordoñez O., Díaz G., Roa L. Valor nutricional de forraje de pízamo (Erythrina glauca) ensilado con diferentes niveles de melaza y harina de arroz. Rev. Sist. de Prod. Agroecol., 1: 1: 32-46. 2010.

13. Orskov R., Hovell O. Uso de la técnica de bolsa de nylon para la evaluación de alimentos. Rev Producción Animal Tropical, 213-233. 1982.

14. Owens F. N., Goetsch L. A. Ruminal fermentation. En: Church D. C. (Ed) Rumiant animal, digestive physiology and nutrition. Prentice hall. New Jersey. p. 195-224. 1988.

15. Roa M. L., Céspedes D., Muñoz J. Evaluación nutricional de tres especies de árboles forrajeros en bovinos fistulados en el pie de monte llanero. Rev ACOVEZ, 24: 14-18. 1999.

16. Roa M. L., Galeano, J. R., Muñoz J. Suplementación de vacas doble propósito con Morus alba y Erythrina glauca en época seca en subregión del pie de monte Llanero. Rev. Orinoquia. 6: 1: 70-82. 2002.

17. Roa L. Contenido nutricional de forrajes y alimentos para animales. Universidad de los Llanos, Escuela de Medicinas veterinaria y Zootecnia. Villavicencio, Colombia. p. 38-54. 2004.

18. Rosales M. Efectos asociativos in vitro de mezclas de forrajes arbóreos para rumiantes. $\quad$ p. 1-10. 1999. Disponible En: http://cipav.org.co/redagrofor/memorias99/Memorias.htm. 
19. Russo R. Erythrina: Un género versátil en sistemas agroforestales; revisión bibliográfica. Turrialba. Costa Rica. p. 10-15. 1981.

20. Rossi C. A., Torrá E., González G. L., Lacarra H., Pereyra A. M. Contenido de la proteína bruta de las hojas de Sauce (Salix sp.) y Álamo (Populus sp.) en un sistema silvopastoril del delta del Paraná. Asociación Argentina de producción animal. 2005. Disponible En: http://www.aapa.org.ar/congr ant

21. Van Soest P. J., Robertson J. B., Lewis B. A. Methods for dietary fiber, neutral detergent fiber, and nonstarch polysaccharides in relation to animal nutrition. $J$ Dairy Sci. 74 (10): 3583-97. 1991. 